\title{
A simple model based on wound size and duration predicted healing of venous leg ulcers at 24 weeks
}

Margolis DJ, Berlin JA, Strom BL. Which venous leg ulcers will heal with limb compression bandages? Am J Med 2000

Jul;109:15-9.

\section{QUESTION: In patients with venous leg ulcers, can a simple prediction rule be derived to identify those patients who will heal within 24 weeks with the use of limb compression bandages?}

\author{
Design \\ 2 cohort studies-1 each for model derivation and \\ validation.
}

\section{Settings}

Derivation was done in a retrospective cohort from a cutaneous ulcer centre in a university hospital in Philadelphia, Pennsylvania, United States. Validation was done in $>20$ US clinical sites.

\section{Patients}

260 patients (mean age 66 y, $61 \%$ women, $62 \%$ white) were in the derivation cohort, and 219 patients (mean age $62 \mathrm{y}, 51 \%$ women, $70 \%$ white) were in the validation cohort. Inclusion criteria for the derivation set were venous leg ulcer in the area from mid-calf to 1 inch below the malleolus, past or current history of lower leg edema that had improved with raising the leg, and other venous diseases. Exclusion criteria were ischaemic leg, life expectancy $\leqslant 24$ weeks, recent use of immunosuppressive agents, or history of cutaneous vasculitis or neutrophilic dermatoses. 1 ulcer per person was randomly chosen for study. Multilayered compression bandages were used and changed weekly. Patients who did not complete treatment were considered to have ulcers that were not healed.

\section{Description of prediction guide}

Prognostic factors used in derivation were race, ulcer area, self reported duration, ankle-brachial index, number of ulcers, inability to walk one block, history of debridement, $>50 \%$ of ulcer covered with fibrin, lipodermatosclerosis, and undermined ulcer margin. After multivariate analysis, a model was derived with 1 point each for ulcers $>5 \mathrm{~cm}^{2}$ and $>6$ months old.

\section{Main outcome measure}

Healed ulcers within 24 weeks using the definition of the Wound Healing Society.

\section{Main results}

By 24 weeks, ulcers had healed in $65 \%$ of patients in the derivation cohort and in $56 \%$ of patients in the validation cohort. The table shows percentages of wounds healed for scores of 0,1 , and 2 . The model correctly discriminated between ulcers that healed and those that did not within 24 weeks $87 \%$ of the time (area under the receiver operating characteristic curve 0.87 , $95 \%$ CI 0.83 to 0.91 ).

\section{Conclusion}

A simple prediction rule using ulcer area and duration identified venous leg ulcers that were healed within 24 weeks using limb compression bandages.

Prediction of percentage of venous leg ulcers healed by 24 weeks in derivation and validation cohorts*

\begin{tabular}{lll} 
Score & $\begin{array}{l}\text { Derivation cohort } \\
(95 \% \mathrm{Cl})\end{array}$ & $\begin{array}{l}\text { Validation cohort } \\
\text { (Cl) }\end{array}$ \\
\hline 0 & $93 \%(87$ to 97$)$ & $95 \%(75$ to 99$)$ \\
\hline 1 & $65 \%(54$ to 76$)$ & $73 \%(62$ to 83$)$ \\
\hline 2 & $13 \%(6$ to 24$)$ & $37 \%(28$ to 45$)$ \\
\hline
\end{tabular}

* 1 point if ulcer is $>5 \mathrm{~cm}^{2}$ and 1 point if ulcer is $>6$ months old.

\section{COMMENTARY}

Although venous leg ulcers occur in up to $1 \%$ of the elderly population, limited evidence is available on prognostic factors that influence healing time. ${ }^{1}$ Margolis $e t$ al have developed a simple rule for predicting which venous leg ulcers are likely to heal within 24 weeks of treatment with limb compression bandages and suggest that identification of "hard to heal" ulcers could guide referral to specialist services.

The participants were all from the US and were treated with compression bandages. The findings, however, may differ in other settings.

The results are relevant to people who care for patients with leg ulcers, manage leg ulcer services, or research leg ulcer treatments. The findings allow us to provide patients with information on the likelihood that their ulcers will heal within 24 weeks simply on the basis of ulcer duration and size. The study shows that ulcers of $\leqslant 6$ months of duration and $\leqslant 5 \mathrm{~cm}^{2}$ in area that are treated with compression will probably heal within 24 weeks. Conversely, ulcers of $>6$ months of duration and $>5 \mathrm{~cm}^{2}$ in area are unlikely to heal within 24 weeks. Complex measurement techniques are unnecessary to ascertain whether an ulcer area is $>5 \mathrm{~cm}^{2}$, as the authors also show that ulcer length multiplied by ulcer width is an adequate measure of area.

Ulcer duration and area are key characteristics that should be evenly distributed between treatment groups in randomised trials. Future trials should use such strategies as stratified randomisation to ensure that even distribution occurs, and researchers should report the distribution of these variables between groups at baseline.

The prediction rule applies to individual ulcers rather than to individual patients who may have numerous ulcers. This means that the results might not help to predict whether a patient will be ulcer free in 24 weeks, probably the most important outcome for patients. By using this approach, those patients likely to have protracted healing times can be identified and may benefit from the use of other treatments in addition to compression, such as pentoxifylline. ${ }^{2}$

E Andrea Nelson, RN University of York York, UK

Callam M. Prevalence of chronic leg ulceration and severe chronic venous disease in western countries. Phlebology 1992;7(suppl 1):6-12.

2 Jull AB, Waters J, Arroll B. Oral pentoxifylline for treatment of venous leg ulcers. Cochrane Database Syst Rev 2000;(2):CD001733. 